\title{
OPEN Impact of antibiotic usage on extended-spectrum $\beta$-lactamase producing Escherichia coli prevalence
}

\author{
Jeong Yeon Kim ${ }^{1,6}$, Yunjin Yum ${ }^{2,6}$, Hyung Joon Joo ${ }^{3,4}$, Hyonggin $\mathrm{An}^{2,3}$, Young Kyung Yoon ${ }^{1}$, \\ Jong Hun Kim ${ }^{5,6 \bowtie}$ \& Jang Wook Sohn ${ }^{1,3,6 \bowtie ~}$
}

An increase in antibiotic usage is considered to contribute to the emergence of antimicrobial resistance. Although experts are counting on the antimicrobial stewardship programs to reduce antibiotic usage, their effect remains uncertain. In this study, we aimed to evaluate the impact of antibiotic usage and forecast the prevalence of hospital-acquired extended spectrum $\beta$-lactamase (ESBL)_producing Escherichia coli (E. coli) using time-series analysis. Antimicrobial culture information of $E$. coli was obtained using a text processing technique that helped extract free-text electronic health records from standardized data. The antimicrobial use density (AUD) of antibiotics of interest was used to estimate the quarterly antibiotic usage. Transfer function model was applied to forecast relationship between antibiotic usage and ESBL-producing $E$. coli. Of the 1938 hospitalacquired isolates, 831 isolates (42.9\%) were ESBL-producing E. coli. Both the proportion of ESBLproducing $E$. coli and AUD increased over time. The transfer model predicted that ciprofloxacin AUD is related to the proportion of ESBL-producing $E$. coli two quarters later. In conclusion, excessive use of antibiotics was shown to affect the prevalence of resistant organisms in the future. Therefore, the control of antibiotics with antimicrobial stewardship programs should be considered to restrict antimicrobial resistance.

Antimicrobial resistant bacteria are considered as a significant cause of mortality and socioeconomic burden globally. Of all resistant organisms, extended-spectrum $\beta$-lactamase (ESBL)-producing gram-negative bacteria are characterized as the most critical and top priority antimicrobial-resistant pathogens by the World Health Organization $(\mathrm{WHO})^{1}$. Several studies on risk factors of ESBL-producing organism infections indicate that it is strongly associated with prior usage of antibiotics.

Various classes of antibiotics have been described as risk factors for the prevalence of ESBL-producing organisms, including cephalosporins, carbapenems, and trimethoprim/sulfamethoxazole ${ }^{2,3}$. These previous studies were based on the concept that using antimicrobial drug results in selective pressure toward the emergence of resistance ${ }^{4}$. However, the direct consequence of specific antibiotic usage on antimicrobial resistance has not been established yet ${ }^{5,6}$.

An antibiotic or antimicrobial stewardship program is a coordinated intervention established by healthcare professionals to select the most appropriate antibiotic, duration, dose, and route of administration for a given patient with an intention to minimize the impact on antibiotic resistance ${ }^{7}$. Most tertiary hospitals are implementing antibiotic stewardship programs even if there is insufficient evidence regarding any relationship between selective antibiotic consumption and antimicrobial resistance. There have been only a few attempts to assess the impact of antibiotic stewardship program using the in-hospital data ${ }^{8,9}$.

One of the challenges in elucidating this relationship is that the process of analyzing the antimicrobial data and measurement of the level of prior usage of antibiotic is complicated ${ }^{10}$. The evaluation of time-dependent

\footnotetext{
${ }^{1}$ Division of Infectious Diseases, Department of Internal Medicine, Korea University College of Medicine, Seoul, Republic of Korea. ${ }^{2}$ Department of Biostatistics, Korea University College of Medicine, Seoul, Republic of Korea. ${ }^{3}$ Korea University Research Institute for Medical Bigdata Science, Korea University, Seoul, Republic of Korea. ${ }^{4}$ Department of Cardiology, Cardiovascular Center, Korea University College of Medicine, Seoul, Republic of Korea. ${ }^{5}$ Division of Infectious Diseases, Department of Internal Medicine, CHA Bundang Medical Center, CHA University, Seongnam, Republic of Korea. ${ }^{6}$ These authors contributed equally: Jeong Yeon Kim, Yunjin Yum, Jong Hun Kim and Jang Wook Sohn. ${ }^{\circledR}$ email: smonti1976@hotmail.com; jwsohn@korea.ac.kr
} 


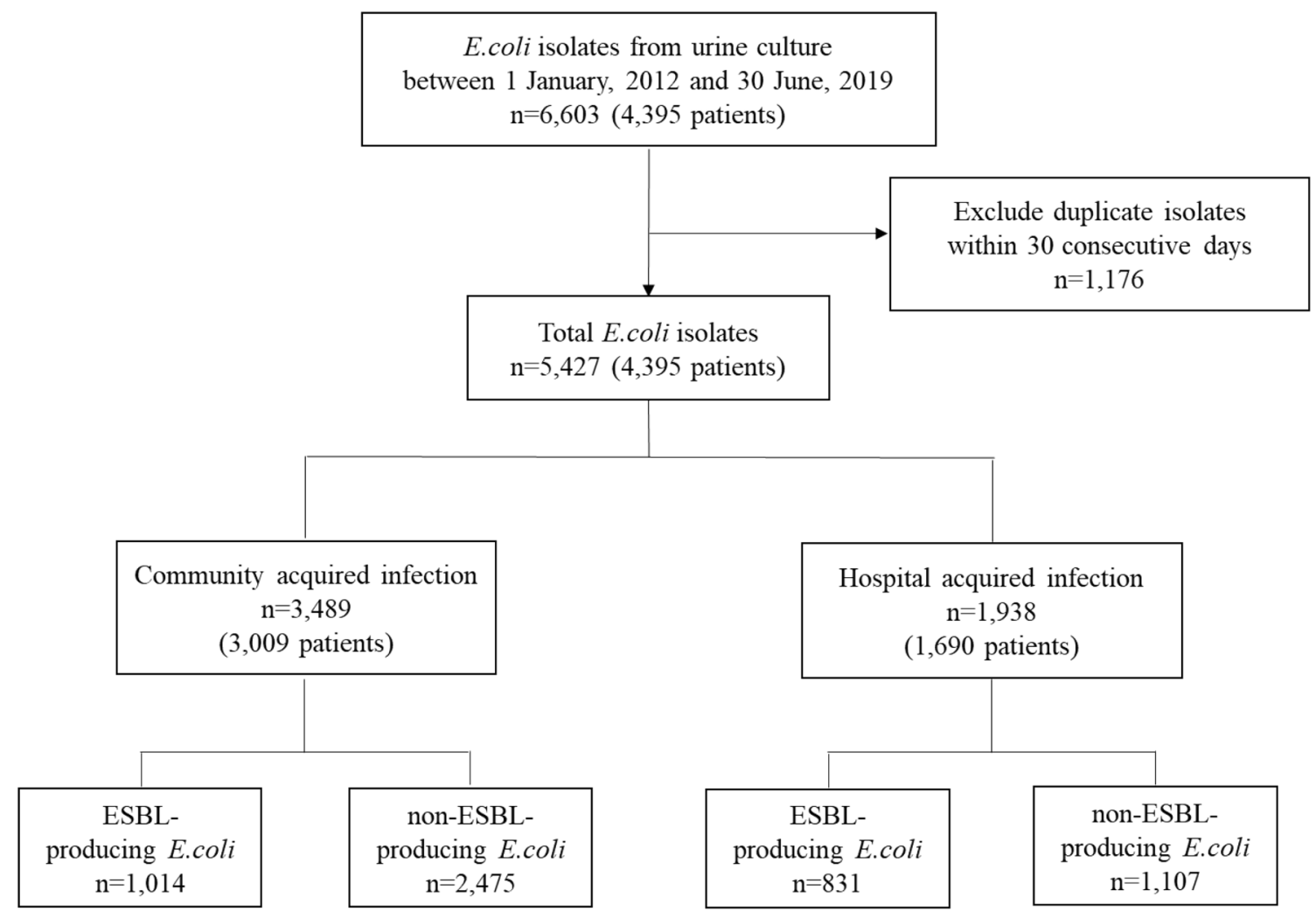

Figure 1. Flow diagram depicting the study population.

variables requires the establishment of a complex analysis system ${ }^{11}$. However, the implementation of big data analysis models on hospital electronic health record (EHR) systems made this easier. The development of a testprocessing algorithm that can convert text-wise EHR data to standardized data has made it possible to process the immense data, including antimicrobial resistance phenotypes.

In the present study, we aimed to investigate the relationship between the volume of antibiotic consumption and proportion of hospital-acquired ESBL-producing Escherichia coli (E. coli) detected in urine culture in a tertiary hospital where an antibiotic stewardship program is implemented on some of the restricted antibiotics. Time-series correlating the antibiotic consumption and prevalence of urinary ESBL-producing E. coli were hypothesized. Finally, time-dependent models of the effects of antibiotic consumption on the prevalence of urinary ESBL-producing E. coli were statistically proposed.

\section{Results}

A total of 6603 urine cultures in which $E$. coli was identified were collected from 4395 patients who visited the emergency department or in-patient unit between 1 January 2012 and 30 June 2019. Duplicative isolates obtained from urine cultures that were conducted within a span of 30 consecutive days were regarded as one result. Among 4395 patients, $81.7 \%(\mathrm{n}=3589)$ were female. The average age of females and males was $67.6 \pm 16.1$ and $68.4 \pm 14.4$, respectively. We defined cases as community-onset cases when urine culture was collected in $48 \mathrm{~h}$ of admission and as hospital-acquired cases when the collection time exceeded $48 \mathrm{~h}$. Among 5427 isolates, $64.3 \%$ $(n=3489)$ were considered as community-onset, and the average time difference between specimen collection and admission for these cases was $6.1 \pm 12.5 \mathrm{~h}$. For hospital-acquired isolates, the average time difference was $561.2 \pm 1497.1 \mathrm{~h}$. Moreover, among hospital-acquired isolates, $42.9 \%(\mathrm{n}=831)$ were identified as ESBL-producing E. coli, while $29.1 \%(\mathrm{n}=1014)$ of community-onset isolates were ESBL-producing E. coli.

To establish the relationship between antibiotic consumption volume and the prevalence of hospital-acquired ESBL-producing E. coli isolates, trend and time-series analyses using the ARIMA model were conducted as described below (Fig. 1).

ESBL-producing $E$. coli proportion and antibiotic usage trend. The proportion of ESBL-producing $E$. coli increased over time $(\beta=0.0062, P<0.0001)$. Similarly, antimicrobial use density (AUD) of ciprofloxacin $(\beta=1383.6622, P=0.0026)$, cefepime $(\beta=961.8701, P<0.0001)$, piperacillin-tazobactam $(\beta=2457.3924, P<0.0001)$, and third-generation cephalosporine $(\beta=3035.1029, P<0.0001)$ consumption showed an increasing trend over time (Fig. 2).

Correlation between ESBL-producing $E$. coli proportion and antibiotic usage. We identified a correlation between the proportion of ESBL-producing E. coli and antibiotic usage. The proportion of ESBL- 
(A)

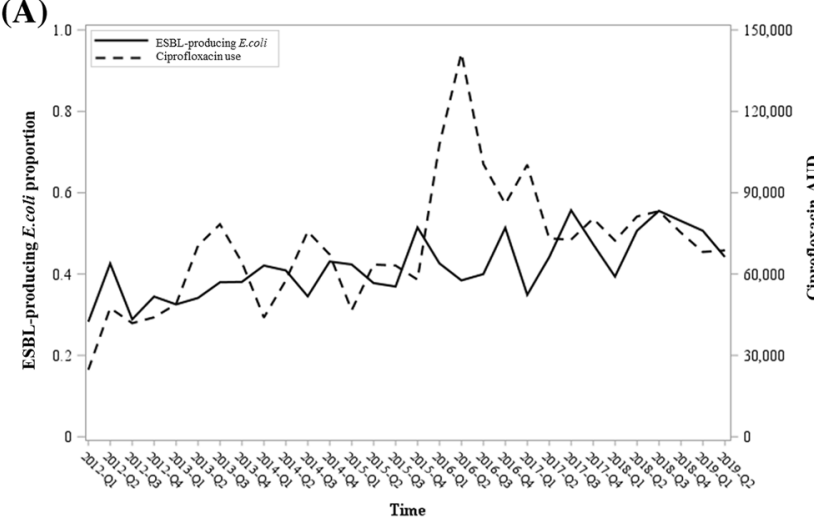

(C)

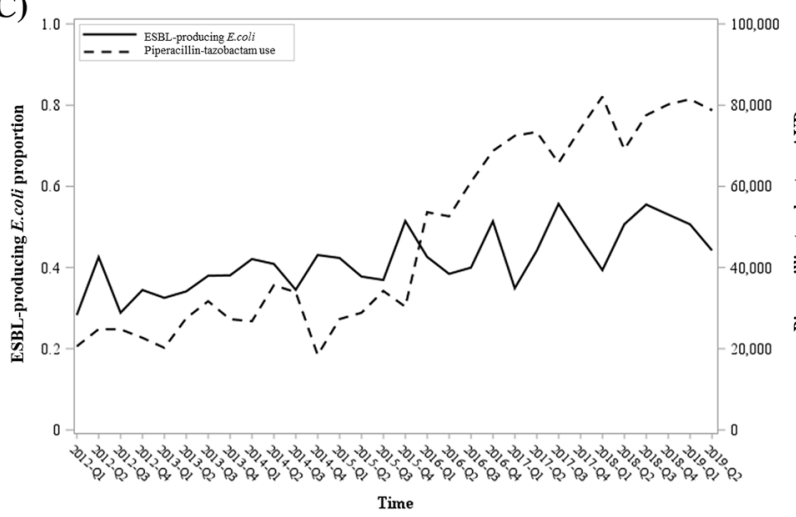

(B)

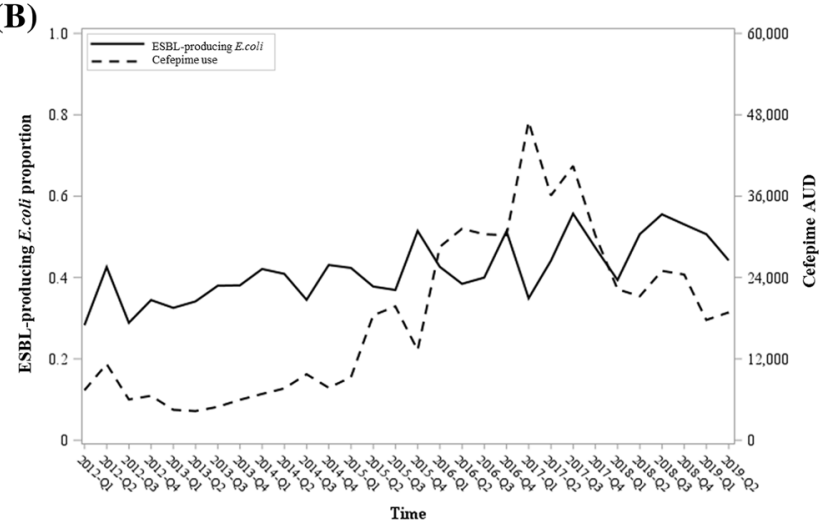

(D)

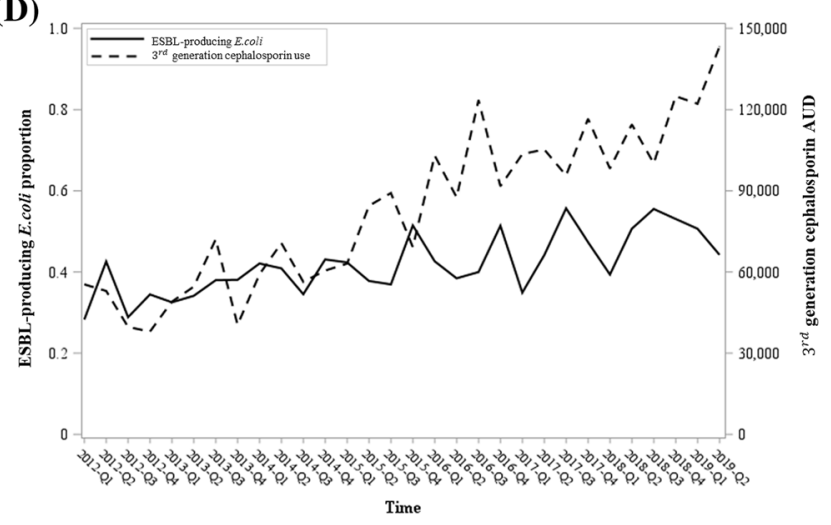

Figure 2. Time-series plot of ESBL-producing E. coli proportion and AUD from 1 January 2012 to 30 June 2019. AUD of (A) ciprofloxacin, (B) cefepime, (C) piperacillin-tazobactam and (D) third-generation cephalosporin.

\begin{tabular}{|l|l|l|l|l|l|l|}
\hline Parameter & Estimate & SE & $\boldsymbol{t}$ value & $\boldsymbol{p}$ value & Lag & Shift \\
\hline AR1,1 & 0.9721 & 0.0361 & 26.91 & $<0.0001$ & 1 & 0 \\
\hline NUM1 & 0.0000024 & 0.0000006 & 4 & $<0.0001$ & 0 & 2 \\
\hline
\end{tabular}

Table 1. ESBL-producing E. coli proportion and ciprofloxacin usage parameter estimates based on the transfer function model. The autoregressive parameter was labelled as AR1,1: representing past value of ESBLproducing E. coli proportion. The numerator term was labelled as NUM1: ciprofloxacin usage. SE, standard error.

producing E. coli was associated with ciprofloxacin usage two quarters earlier. Conversely, there was no correlation between the proportion of ESBL-producing E. coli and the usage of cefepime, piperacillin-tazobactam, and/ or third-generation cephalosporin. Therefore, we used ciprofloxacin usage to build a transfer function model (Fig. S1).

Ciprofloxacin usage and ESBL-producing E. coli proportion. The transfer function model was used to elucidate the relationship between ESBL-producing E. coli proportion and ciprofloxacin usage. Table 1 presents the transfer function model for ESBL-producing E. coli and ciprofloxacin AUD. The results show that an increase by 1 unit of ciprofloxacin usage is related to an increase in the proportion of ESBL-producing E. coli two quarters later by 0.0000024 , and the proportion of ESBL-producing E. coli in the previous quarter was related to proportion of ESBL-producing E. coli in the present quarter. The transfer function model for the ESBL-producing E. coli proportion $\left(Y_{t}\right)$ and ciprofloxacin usage $\left(X_{t}\right)$ according to the Table 1 can be expressed as follows:

$$
Y_{t}=0.0000024 X_{t-2}+\frac{a_{t}}{1-0.9721 B}
$$

where $a_{t}$ denotes white noise and $B$ denotes back shift.

The residual plots of the autocorrelation function (ACF) and partial autocorrelation function (PACF) showed that the assumptions of the transfer function model are acceptable, implying that the residuals correspond to the white noise (Fig. S2). The Akaike information criterion (AIC) and Schwarz-Bayesian information criterion 


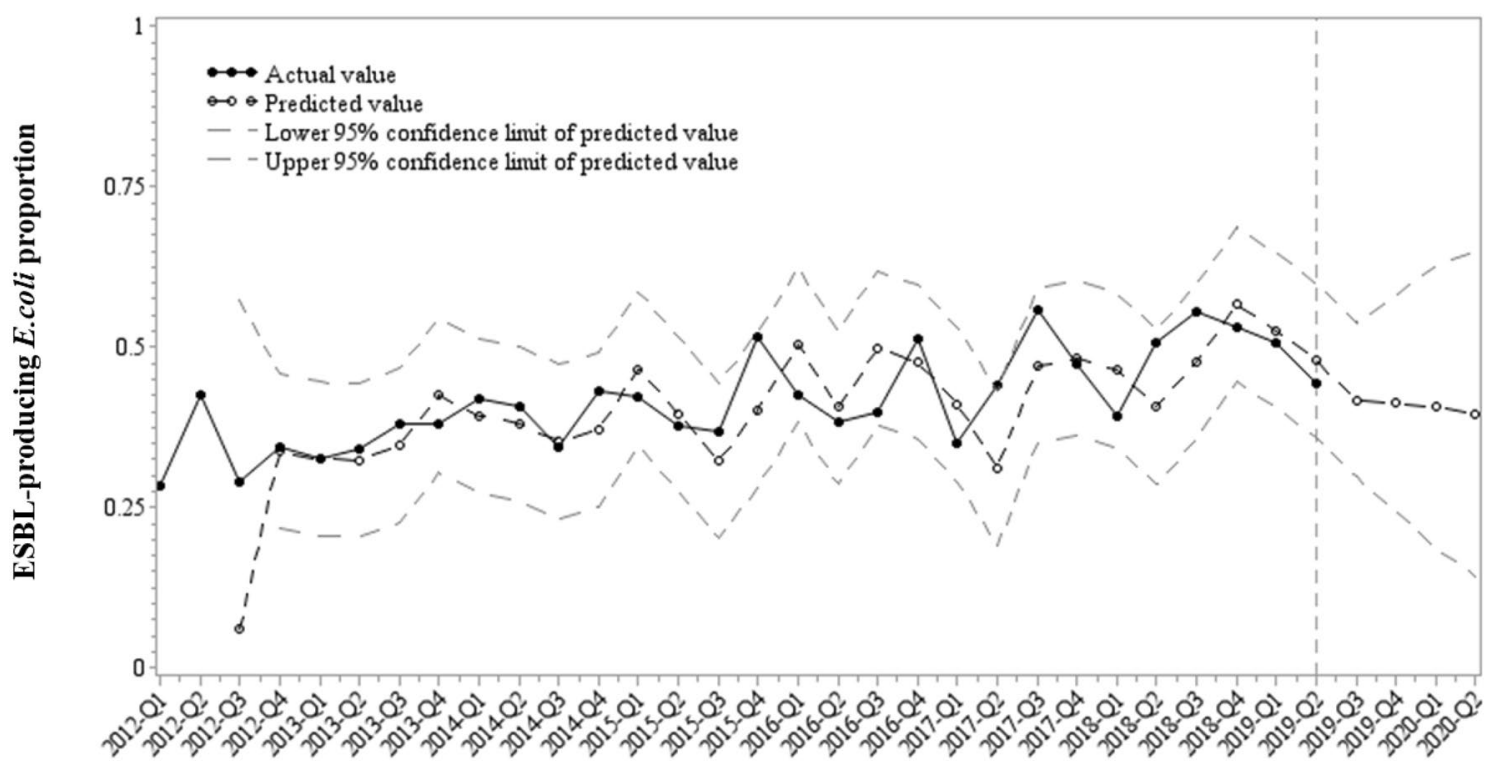

Time

Figure 3. Predicted and observed ESBL-producing E. coli proportion upon ciprofloxacin usage.

(SBC) were -71.9579 and -69.2935 , respectively, and the mean absolute percentage error (MAPE) value was 13.4336. The ESBL-producing E. coli proportion predictions for the next four quarters were $0.4182,0.4124$, 0.4065 , and 0.3970 (Fig. 3).

\section{Discussion}

The main accomplishments of the present study are as follows: (1) development of a unique method to process text-wise EHR data into standardized data made it possible to set up a model of large sets of data in a time-series; and (2) this time-dependent model demonstrated the volume of ciprofloxacin consumption in the six months prior to the prediction, predicts the proportion of ESBL-producing E. coli.

To elucidate the association between antibiotic consumption and related antimicrobial resistance, in the present study, a new database of antibiotic orders and bacterial culture reports, which were extracted and transformed from EHRs between 2012 and 2019, was established. Explorative data analysis revealed that the quarterly proportion of ESBL-producing E. coli in urine culture steadily increased over time. AUD of antibiotics of interest also showed an increasing trend over time. Among them, AUD of third-generation cephalosporin increased most exponentially with time and ranked the highest among all tested antibiotics. This in-hospital data correlated with the national reimbursement data demonstrating the total antibiotic consumption nationally, which also showed a significant increase in the trends of third-generation cephalosporin consumption in in-hospital settings ${ }^{12}$.

Time-dependent model of our data predicted that the detection of ESBL-producing E. coli in urine culture increased with the consumption of ciprofloxacin six months earlier than the present stipulated time. A previous study that was carried out in our center 12 years ago also showed that the proportion of ciprofloxacin-resistant E. coli was associated with consumption level in the 2 months prior to the measurement ${ }^{8}$. Although many ESBLproducing E. coli were ciprofloxacin resistant, their resistance remained in steady manner throughout the study period, while ESBL-producing E. coli proportion trend was increasing (Fig. S3). The present study revealed that the consumption of certain antibiotics increased not only its specific antibiotic resistance but also the expression of ESBL phenotype, further widening the spectrum of antimicrobial resistance. These results suggest that the level of in-hospital antibiotic usage is directly associated with an increase in antimicrobial resistance in nosocomial settings. Conversely, the restriction of using specific antibiotics may lead to a decrease in resistance to pathogens, which can be successfully implemented with antibiotic stewardship programs. Currently, ciprofloxacin in our center is not included in the in-hospital antibiotic stewardship program and was freely prescribed by physicians without any restrictions or recommendations. However, an indiscreet usage of antibiotics should be avoided, restriction on antibiotic usage should also be implemented cautiously to avoid adverse effects, such as increased mortality or longer duration of hospital stay. Despite reducing antibiotic consumption, antimicrobial resistance might still persist due to its relatively low fitness $\operatorname{cost}^{13}$. Notably, Lozano et al. ${ }^{14}$ identified the minimum threshold of fluoroquinolone usage in lowering ESBL-producing E. coli below 100 DDDs per 1000 occupied bed days using a nonlinear time-series analysis, which our center far exceeds.

Other studies have shown a strong relationship between the usage of third-generation cephalosporin and expression of ESBL phenotype ${ }^{3,15,16}$. Tacconelli et al. $^{3}$ reported that cephalosporin monotherapy ranked first among all antibiotics in promoting colonization of ESBL gram-negative bacteria in rectal swabs through analyzing the patient-level data with machine learning methods. However, in our study, this correlation was not evident. This was an unexpected result considering that third-generation cephalosporin was the most frequently used 
antibiotic in hospitals. One way to explain this result is that third-generation cephalosporin has been managed by antimicrobial stewardship program since 2002 in our center. It was prescribed only under the authorization of antibiotic use program by infectious disease department specialists to minimize its unnecessary usage and misuse. This situation in the center might have led to the above results, implying that antimicrobial stewardship program may exert antibiotic selective pressure. Although there are controversial data on the effect of antimicrobial stewardship program on antibiotic resistance ${ }^{9}$, similar results have been reported in a recent study conducted by Hwang et al. ${ }^{17}$ using interrupted time-series analysis, which showed a decrease in the antimicrobial resistance rate of Staphylococcus aureus and Pseudomonas aeruginosa when antimicrobial stewardship intervention was implemented. Therefore, using broader classes of restricted antibiotics, such as fluoroquinolones, in antimicrobial stewardship programs should be considered to restrict ESBL-producing E. coli.

Regarding cefepime, it was difficult to obtain a significant relationship between AUD and the proportion of ESBL-producing E. coli. The consumption of piperacillin-tazobactam and proportion of ESBL-producing $E$. coli were correlated with a time interval of 33 months (lag of 11), which was very difficult to interpret clinically. Cefepime usage did not show a significant correlation with ESBL-producing E. coli. Cefepime and piperacillintazobactam antibiotics were already included in the hospital antibiotic stewardship program and were regulated along with third-generation cephalosporin. In particular, our center strongly recommended the usage of piperacillin-tazobactam and cefepime as selective antibiotics for ESBL-producing urinary tract infections, rather than carbapenem, based on the carbapenem-sparing strategy. This means that the level of piperacillin-tazobactam and cefepime usage in hospitals is dynamically influenced by the current proportion of ESBL-producing $E$. coli. This relationship might have acted as a confounding factor in the association of the antibiotics with ESBLproducing E. coli.

This study has some limitations. First, the time-series transfer function model reflects the data collected at different time points. Therefore, other clinically relevant characteristics were not included in the model, such as sex, co-morbidity, and invasive procedure such as indwelling catheter. Further investigation on predicting patient-level ESBL-producing organism proportions should be conducted using other models. Second, expansion of ESBL-producing E. coli due to nosocomial transmission or in-hospital outbreaks instead of antibiotic selective pressure was not taken into consideration. This clinical situation may have led to misinterpretation. Third, the transfer function model built using ciprofloxacin showed a slightly high MAPE value of 13.4336, indicating that the forecast can be off by $13.4336 \%$. However, by using longer time-series data and taking into consideration other risk factors, forecasting measurement error can be reduced ${ }^{18}$. Fourth, the size of the hospital might be a potential confounding factor. The effect of antimicrobial consumption on the prevalence of ESBL-producing $E$. coli might be different in small- or large-sized hospitals. Therefore, a multi-center study including both small and large hospitals should to be performed in the future.

Despite these limitations, the present study demonstrates the relationship between antibiotic consumption and the prevalence of antimicrobial resistance. Efforts to reduce inappropriate antibiotic usage, especially of fluoroquinolones in clinical practice, should be made in hospitals through antimicrobial stewardship programs. Moreover, the development of algorithms for extracting the text-wise data from electronic health records provides a much easier approach for the use of antimicrobial data from other hospitals in addition to that from our center. Further research on other data sets may provide insights into additional relationships between AUD and antimicrobial resistance by using a transfer function model as well as other methods, such as other mathematical models or machine learning.

\section{Methods}

Data approval. The study protocol was approved by the Institutional Review Board of Korea University Anam Hospital (IRB No. 2019AN0228). Written informed consent was waived by the Institutional Review Board of Korea University Anam Hospital because of the retrospective study design with minimal risk to participants. The study complied with the principles of the Declaration of Helsinki.

Bacterial culture report data processing. The bacterial culture reports from 1 January 2012 to 30 June 2019 were extracted from the electronic health record (EHR) database. Since the bacterial culture reports were in a semi-standardized free-text format, detailed standardized data were further extracted using text processing techniques, including regular expressions. The names of the bacterial organisms and antimicrobials were standardized.

Microbiological evaluation. Escherichia coli in the urine culture specimen was identified using the VITEK II (bioMèrieux, Hazelwood, MO, USA) system and matrix-assisted laser desorption/ionization-time of flight (MALDI-TOF) mass spectrometry (Bruker, Coventry, UK). Antibiotic susceptibility testing was performed using the VITEK II (bioMèrieux, Hazelwood, MO, USA) system or MicroScan WalkAway 96 plus (Siemens Healthcare Diagnostics Inc., CA, USA) system following the Clinical and Laboratory Standards Institute (CLSI) guidelines ${ }^{19}$. Phenotypic screening for ESBL production was performed using the VITEK II (bioMèrieux, Hazelwood, MO, USA) system.

Antimicrobial consumption data. Pharmacological data were determined according to the Anatomical Therapeutic Chemical (ATC) classification with the defined daily dose (DDD) as a unit of measure, as recommended by the WHO Collaborating Centre for Drug Statistics Methodology ${ }^{20}$. DDD is defined as the assumed average maintenance dose per day for a drug used for its main indication in adults. Data of five classes of antibiotics of interest (ciprofloxacin, third-generation cephalosporin including ceftriaxone, cefotaxime, and ceftazidime, cefepime, trimethoprim-sulfamethoxazole, and piperacillin-tazobactam) were extracted from EHR 
in grams and international unit form and converted into DDD. AUD was used to estimate the quarterly usage of antibiotics. AUD was calculated as follows:

$$
\text { AUD }=\left[\frac{\text { total antibiotic consumption amount }(\mathrm{mg})}{\text { DDDs }(\mathrm{mg}) \times \text { patients per year }}\right] \times 1000
$$

Statistical analysis. Using linear regression, quarterly trends in the proportion of ESBL-producing E. coli and antibiotic usage were verified over time. We applied a transfer function model to investigate the association between antibiotic consumption and the proportion of ESBL-producing E. coli. The modeling was performed following three procedures: (1) building an adequate ARIMA model for two time-series, antibiotic use, and ESBL-producing E. coli proportion; (2) identifying the cross-correlation between two time-series to determine the systematic part of the transfer function; and (3) diagnostic checking of the chosen model: autocorrelation function $(\mathrm{ACF})$ and partial correlation function (PACF) of residuals were identified to determine whether they were contributing to the white noise. The AIC and SBC were computed for evaluating goodness of fit and mean absolute percentage error (MAPE) for the measurement of forecast error.

$$
\text { MAPE }=\frac{1}{N} \sum_{t}^{N} \frac{\left|Y_{t}-\widehat{Y}_{t}\right|}{Y_{t}} \times 100 .
$$

where $Y_{t}$ denotes the observed ESBL-producing $E$. coli proportion at month $\mathrm{t}$ and $\widehat{Y}_{t}$ denotes the forecasted ESBLproducing E. coli proportion at month t. All statistical analyses were performed using SAS 9.4 (SAS Institute Inc., Cary, NC, USA) program.

\section{Data availability}

The datasets used and analyzed during the present study can be available form the corresponding author, Jang Wook Sohn, upon reasonable request.

Received: 20 January 2021; Accepted: 17 May 2021

Published online: 22 June 2021

\section{References}

1. Tacconelli, E. et al. Discovery, research, and development of new antibiotics: the WHO priority list of antibiotic-resistant bacteria and tuberculosis. Lancet Infect. Dis. 18, 318-327. https://doi.org/10.1016/S1473-3099(17)30753-3 (2018).

2. Zhu, F. H. et al. Risk factors for community acquired urinary tract infections caused by extended spectrum $\beta$-lactamase (ESBL) producing Escherichia coli in children: a case control study. Infect. Dis. 51, 802-809. https://doi.org/10.1080/23744235.2019.16541 27 (2019).

3. Tacconelli, E. et al. Estimating the association between antibiotic exposure and colonization with extended-spectrum beta-lactamase-producing Gram-negative bacteria using machine learning methods: a multicentre, prospective cohort study. Clin. Microbiol. Infect. 26, 87-94. https://doi.org/10.1016/j.cmi.2019.05.013 (2020).

4. Schwartz, B., Bell, D. M. \& Hughes, J. M. Preventing the emergence of antimicrobial resistance: a call for action by clinicians, public health officials, and patients. JAMA 278, 944-945. https://doi.org/10.1001/jama.1997.03550110082041 (1997).

5. Murray, M. T., Beauchemin, M. P., Neu, N. \& Larson, E. L. Prior antibiotic use and acquisition of multidrug-resistant organisms in hospitalized children: A systematic review. Infect. Control Hosp. Epidemiol. 40, 1107-1115. https://doi.org/10.1017/ice.2019. 215 (2019)

6. Lee, E. J., Lee, G., Park, J., Kim, D.-S. \& Ahn, H. S. Analysis of factors affecting antibiotic use at hospitals and clinics based on the defined daily dose. J. Korean Med. Assoc. 61, 687-698. https://doi.org/10.5124/jkma.2018.61.11.687 (2018).

7. Dyar, O. J., Huttner, B., Schouten, J., Pulcini, C. \& Esgap,. What is antimicrobial stewardship?. Clin. Microbiol. Infect. 23, $793-798$. https://doi.org/10.1016/j.cmi.2017.08.026 (2017).

8. Yoon, Y. K. et al. Surveillance of antimicrobial use and antimicrobial resistance. J. Infect. Chemother. 40, 93-101. https://doi.org/ 10.3947/ic.2008.40.2.93 (2008).

9. Kim, B. N., Kim, H. B. \& Oh, M. D. Antibiotic control policies in South Korea, 2000-2013. Infect. Chemother. 48, 151-159. https:// doi.org/10.3947/ic.2016.48.3.151 (2016).

10. Hyle, E. P., Bilker, W. B., Gasink, L. B. \& Lautenbach, E. Impact of different methods for describing the extent of prior antibiotic exposure on the association between antibiotic use and antibiotic-resistant infection. Infect. Control Hosp. Epidemiol. 28, 647-654. https://doi.org/10.1086/516798 (2007).

11. Arepyeva, M. A. et al. A mathematical model for predicting the development of bacterial resistance based on the relationship between the level of antimicrobial resistance and the volume of antibiotic consumption. J. Glob. Antimicrob. Resist. 8, 148-156. https://doi.org/10.1016/j.jgar.2016.11.010(2017).

12. Yoon, Y. K. et al. Trends of antibiotic consumption in Korea according to national reimbursement data (2008-2012): A populationbased epidemiologic study. Medicine (Baltimore) 94, e2100. https://doi.org/10.1097/MD.0000000000002100 (2015).

13. Andersson, D. I. \& Hughes, D. Antibiotic resistance and its cost: is it possible to reverse resistance?. Nat. Rev. Microbiol. 8, 260-271. https://doi.org/10.1038/nrmicro2319 (2010).

14. Lopez-Lozano, J. M. et al. A nonlinear time-series analysis approach to identify thresholds in associations between population antibiotic use and rates of resistance. Nat. Microbiol. 4, 1160-1172. https://doi.org/10.1038/s41564-019-0410-0 (2019).

15. Otter, J. A. et al. Individual- and community-level risk factors for ESBL Enterobacteriaceae colonization identified by universal admission screening in London. Clin. Microbiol. Infect. 25, 1259-1265. https://doi.org/10.1016/j.cmi.2019.02.026 (2019).

16. Nakai, H. et al. Prevalence and risk factors of infections caused by extended-spectrum $\beta$-lactamase (ESBL)-producing Enterobacteriaceae. J. Infect. Chemother. 22, 319-326. https://doi.org/10.1016/j.jiac.2016.02.004 (2016).

17. Hwang, H. \& Kim, B. Impact of an infectious diseases specialist-led antimicrobial stewardship programmes on antibiotic use and antimicrobial resistance in a large Korean hospital. Sci. Rep. 8, 14757. https://doi.org/10.1038/s41598-018-33201-8 (2018).

18. Seong, K.-T., Choi, Y.-H., Koo, J.-H. \& Lee, M.-J. Transfer function model forecasting of sea surface temperature at Yeosu in Korean coastal waters. J. Korean Soc. Mar. Environ. Saf. 20, 526-534. https://doi.org/10.7837/kosomes.2014.20.5.526 (2014). 
19. Humphries, R. M. et al. CLSI methods development and standardization working group best practices for evaluation of antimicrobial susceptibility tests. J. Clin. Microbiol. https://doi.org/10.1128/JCM.01934-17 (2018).

20. ATC/DDD Index 2021. https://www.whocc.no/atc_ddd_index/.

\section{Acknowledgements}

This research was supported by a grant received from the Korea Health Technology R\&D Project through the Korea Health Industry Development Institute (KHIDI), funded by the Ministry of Health and Welfare, Republic of Korea (Grant No. HI19C0201) and the MSIT (Ministry of Science and ICT), Korea, under the ICT Creative Consilience program (IITP-2021-2020-0-01819) supervised by the IITP (Institute for Information and Communications Technology Planning and Evaluation).

\section{Author contributions}

H.J., J.H.K., and J.S. designed the study. H.J. and Y.Y. planned the experiments and performed the analyses. J.Y.K. and Y.Y. wrote the manuscript. Y.Y. prepared the figures and tables. H.J., H.A., J.H.K, and J.S. supervised the conceptualization and writing. J.Y.K., Y.Y., H.J., H.A., Y.K.Y., and J.S. contributed to the collection of the data, discussions, and interpretation of the data.

\section{Competing interests}

The authors declare no competing interests.

\section{Additional information}

Supplementary Information The online version contains supplementary material available at https://doi.org/ 10.1038/s41598-021-91332-x.

Correspondence and requests for materials should be addressed to J.H.K. or J.W.S.

Reprints and permissions information is available at www.nature.com/reprints.

Publisher's note Springer Nature remains neutral with regard to jurisdictional claims in published maps and institutional affiliations.

(c) (i) Open Access This article is licensed under a Creative Commons Attribution 4.0 International License, which permits use, sharing, adaptation, distribution and reproduction in any medium or format, as long as you give appropriate credit to the original author(s) and the source, provide a link to the Creative Commons licence, and indicate if changes were made. The images or other third party material in this article are included in the article's Creative Commons licence, unless indicated otherwise in a credit line to the material. If material is not included in the article's Creative Commons licence and your intended use is not permitted by statutory regulation or exceeds the permitted use, you will need to obtain permission directly from the copyright holder. To view a copy of this licence, visit http://creativecommons.org/licenses/by/4.0/.

(C) The Author(s) 2021 